\title{
Static structure factor of capillary waves at large momentum transfer
}

\author{
M. Paulus, ${ }^{1}$ C. Gutt, ${ }^{2}$ and M. Tolan ${ }^{1}$ \\ ${ }^{1}$ Fakultät Physik/DELTA, Technische Universität Dortmund, 44221 Dortmund, Germany \\ ${ }^{2}$ Deutsches Elektronen Synchrotron (HASYLAB), Notkestr. 85, 22607 Hamburg, Germany \\ (Received 5 September 2007; revised manuscript received 31 October 2008; published 11 December 2008)

\begin{abstract}
The x-ray scattering cross section from capillary waves at large values of the momentum transfer parallel to the surface is discussed. In contrast to the conventional approach where only the height-height correlation of the surface is taken into account the full-wave-motion-induced static correlations are embedded in the calculation. This leads to an increase in scattering intensity especially at large wave vector parallel to the sample's surface which has been interpreted as evidence for a wave-vector-dependent surface tension previously. We are discussing under which conditions surface-sensitive x-ray scattering experiments can be used to derive the surface Hamiltonian of capillary waves.
\end{abstract}

DOI: 10.1103/PhysRevB.78.235419

PACS number(s): 61.05.cc, 68.03.-g

\section{INTRODUCTION}

Currently there is considerable interest in small wavelength fluctuations of liquid surfaces. ${ }^{1-4}$ At length scales ranging from micrometers to $100 \mathrm{~nm} \mathrm{x}$-ray scattering experiments proved that continuum hydrodynamics is a valid description of the surface fluctuations. ${ }^{5,6}$ At smaller length scales the continuum theory is expected to break down and details of the molecular interactions will be visible in the capillary-wave fluctuations. Mecke and Dietrich ${ }^{7}$ used density-functional theory to calculate the effective interfacial Hamiltonian $H\left(q_{\|}\right)$for capillary waves at large wave-vector transfers $q_{\|}$parallel to the liquid surface. They predict an effective wavelength-dependent surface tension defined as $\gamma\left(q_{\|}\right)=H\left(q_{\|}\right) / q_{\|}^{2}$ which is a nonmonotonic function of $q_{\|}$reflecting both the intrinsic density profile of the liquid and the interaction potentials.

In order to investigate the Mecke-Dietrich predictions a number of researchers used grazing incidence $\mathrm{x}$-ray diffraction (GID) on liquid surfaces to study capillary-wave excitations at large values of the momentum transfer $\left(q_{\|}\right.$ $>10^{-2} \AA^{-1}$ ) parallel to the surface. ${ }^{1-4}$ In such experiments the incoming $x$-ray beam hits the sample's surface under a grazing angle smaller than the critical angle of external total reflection. This leads to an $x$-ray penetration depth in the sample of approximately $10 \mathrm{~nm}$. Depending on the experimental setup a very large $q_{\|}$range is accessible in such an experiment. In our work scattering out of the scattering plane defined by the surface normal and the incident beam is discussed, leading to wave-vector transfer parallel to the sample surface between $10^{-3} \AA^{-1}$ and approximately $3 \AA^{-1}$. Thus, correlations in the surface near scattering volume in a range between micrometer and angstrom are accessible. The classical view of surface scattering from liquids is that the scattering arises from the density contrast at the liquid-vapor interface. This interface is modulated by capillary waves and the scattering is therefore sensitive to the height-height fluctuations of the interface. Referring to the small-anglescattering expressions of Sinha et al. ${ }^{8}$ the authors in Refs. 1-4 assumed that the scattering function $S^{\prime}(\mathbf{Q})$ is directly proportional to the height-height fluctuation spectrum, i.e., $S^{\prime}(Q) \propto k_{B} T / H\left(q_{\|}\right)\left[T\right.$ is the temperature and $k_{B}$ is the Boltz- mann constant, $\mathbf{Q}=\left(q_{\|}, q_{z}\right)$ with the wave-vector transfer perpendicular to the surface $q_{z}$ ]. Deviations of the scattered intensity from the $1 /\left(\gamma q_{\|}^{2}\right)$ power law have been interpreted as an indication for a wavelength-dependent surface tension. The deduced wavelength-dependent surface tensions have then in turn been used to extract surface interatomic potentials from the $\mathrm{x}$-ray scattering data.

On a microscopic level the small-angle-scattering approach has to break down as the scattering arises from individual molecules within the scattering volume which in the case of capillary waves undergo a circular motion exponentially damped into the liquid material. In this work, we derive expressions for the static structure factor of capillary waves taking the horizontal correlations and also the vertical damping of the correlations into account. We show that at large values of the momentum transfer parallel to the surface the measured signal is not proportional to the height-height fluctuation spectra and thus a priori the surface Hamiltonian is not as easily accessible as anticipated in previous work. It is also shown that the consideration of the complete capillary-wave-motion-induced static correlations in the scattering volume has a significant impact on the theoretical description of scattering intensity, leading to deviations from the $1 /\left(\gamma q_{\|}^{2}\right)$ power law, even if a constant surface tension is considered.

\section{STATIC STRUCTURE FACTOR}

Continuum hydrodynamics describes capillary waves as displacements $\mathbf{u}$ of liquid particles from their respective equilibrium positions $\mathbf{R}$. The equilibrium positions $\mathbf{R}$ describe the disordered liquidlike distribution of particles that would result if a bulk liquid was truncated without capillarywave excitations. The capillary-wave displacements u describe a circular particle motion which is exponentially damped into the liquid material according to $\exp \left(-q_{\|}|z|\right)$ where $z$ represents the coordinate vertical to the surface. In a GID experiment the penetration depth of $\mathrm{x}$ rays is typically $10 \mathrm{~nm}$; thus, as soon as $q_{\|}>0.1 \mathrm{~nm}^{-1}$ only a small portion of the molecules in the illuminated volume participates in the wave motion. ${ }^{9}$ Moreover, as soon as $q_{\|}>q_{z}$ the experiment also projects out the lateral component of the displacement- 
displacement correlations and an increase in scattering intensity is expected. So the conditions of a uniform motion of material within the entire scattering volume and $q_{z}>q_{\|}$ which are needed for applying the theory of Sinha et al. ${ }^{8}$ are not fulfilled in GID experiments at large values of the parallel momentum transfer. (For an extensive overview on how to include surface-scattering geometry into the scattering cross section see Ref. 10.)

In order to calculate the scattering from a liquid surface only fluctuations within the capillary-wave model are taken into account. Thus density-density fluctuations in the bulk liquid are not discussed in this work. We start with the definition of the scattering function in first Born approximation ${ }^{11}$

$$
\begin{aligned}
S(\mathbf{Q}) & =\sum_{m=1}^{N} \sum_{n=1}^{N} f(Q)^{2} e^{-i \mathbf{Q} \cdot\left(\mathbf{R}_{m}-\mathbf{R}_{n}\right)}\left\langle e^{-i \mathbf{Q} \cdot\left(\mathbf{u}_{m}-\mathbf{u}_{n}\right)}\right\rangle \\
& =\sum_{m=1}^{N} \sum_{n=1}^{N} f(Q)^{2} e^{-i \mathbf{Q} \cdot\left(\mathbf{R}_{m}-\mathbf{R}_{n}\right)}\left\langle e^{-i q_{\|} \cdot\left(u_{\|_{m}}-u_{\|_{n}}\right)-i q_{z} \cdot\left(u_{z_{m}}-u_{z_{n}}\right)}\right\rangle,
\end{aligned}
$$

with $\mathbf{Q}=\left(q_{\|}, q_{z}\right) . f(Q)$ and $\mathbf{u}_{m}=\left(u_{\|_{m}}, u_{z_{m}}\right)$ denote the atomic form factor and displacement of the liquid particle, respectively. The summation is over all $N$ molecules in the sample with the lateral component of $\mathbf{R}$ extending from $-\infty$ to $\infty$, while the vertical component of $\mathbf{R}$ extends from $-\infty$ to 0 only, in order to take the presence of the surface into account. Note that in contrast to a crystal the components $\mathbf{R}_{m}$ are statistically distributed representing the molecular structure of the liquid.

The displacement vector has a component parallel and perpendicular to the surface with its amplitude exponentially damped into the liquid material. Using the Baker-Hausdorff theorem ${ }^{11}$ for Gaussian fluctuations $\left\langle e^{a+b}\right\rangle=e^{1 / 2\left(\left\langle a^{2}\right\rangle+\left\langle b^{2}\right\rangle+2\langle a b\rangle\right)}$, $\left\langle a^{2}\right\rangle$ for example can be identified as

$$
\left\langle a^{2}\right\rangle=\left\langle\left[i q_{\|} \cdot\left(u_{\|_{m}}-u_{\|_{n}}\right)\right]^{2}\right\rangle=q_{\|}^{2}\left(-\left\langle u_{\|_{n}}^{2}\right\rangle-\left\langle u_{\|_{m}}^{2}\right\rangle+2\left\langle u_{\|_{m}} u_{\|_{n}}\right\rangle\right)
$$

and

$$
\begin{aligned}
\langle a b\rangle= & \left\langle-q_{\|} q_{z}\left[u_{\|_{n}}-u_{\|_{m}}\right]\left[u_{z_{n}}-u_{z_{m}}\right]\right\rangle=-q_{\|} q_{z}\left[\left\langle u_{\|_{n}} u_{z_{n}}\right\rangle\right. \\
& \left.-\left\langle u_{\|_{n}} u_{z_{m}}\right\rangle-\left\langle u_{\|_{m}} u_{z_{n}}\right\rangle+\left\langle u_{\|_{m}} u_{z_{m}}\right\rangle\right] .
\end{aligned}
$$

The static displacement-displacement correlation functions $C_{\alpha, \beta}\left(z, z^{\prime}, x\right)=\left\langle u_{\alpha}(z, 0) u_{\beta}\left(z^{\prime}, x\right)\right\rangle$ are needed for further treatment and are given for example in Refs. 12-15 from where it follows that $C_{x x}\left(x, z, z^{\prime}\right)=C_{z z}\left(x, z, z^{\prime}\right)$ and $C_{x z}\left(x, z, z^{\prime}\right)$ $+C_{z x}\left(x, z, z^{\prime}\right)=0$. This means that $\langle a b\rangle=0$, which is a direct consequence of circular motion of the volume elements. The correlation function $C_{z z}$ is given by $C_{z z}\left(x, z, z^{\prime}\right)$ $=\frac{k_{B} T}{2 \pi} \int_{q_{\min }}^{q_{\max }} d q q_{\|} J_{0}\left(q_{\|} x\right) / H\left(q_{\|}\right) e^{-q_{\|}\left(z+z^{\prime}\right)}$ with the interfacional Hamiltonian $H\left(q_{\|}\right)$and the Bessel function of the first kind $J_{0}\left(q_{\|} x\right) . q_{\max }$ is defined by the inverse molecular diameter $a$, i.e., $q_{\max }=2 \pi / a$ and $q_{\min }=2 \pi / \xi$ is a cutoff due to the limited coherence length $\xi$ of the photon beam. Thus, the scattering function can be rewritten as

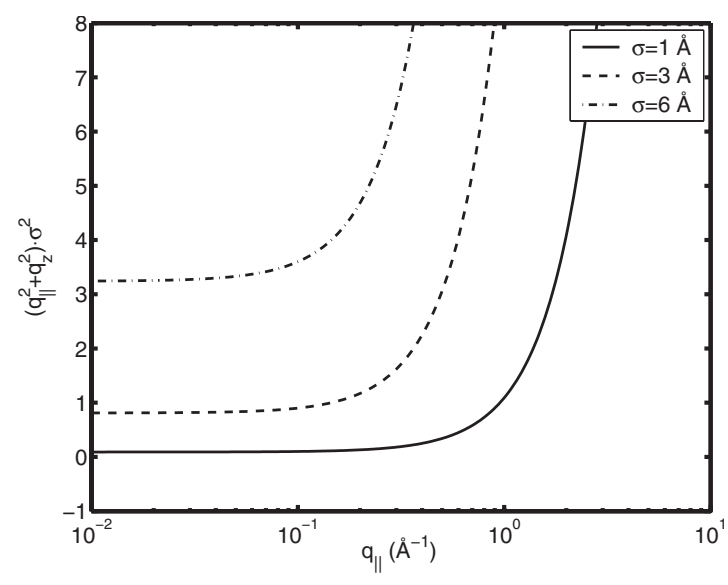

FIG. 1. The product of surface roughness and total momentum transfer as a function of $q_{\|}$given for different surface roughness. The surface roughnesses are typical values for liquid metals (black line), water (dashed), and liquid alkane (dotted).

$$
\begin{aligned}
S(\mathbf{Q})= & \sum_{m, n} f^{2}(Q) e^{-i \mathbf{Q} \cdot\left(\mathbf{R}_{m}-\mathbf{R}_{n}\right)} e^{-q_{z}^{2} / 2\left[C_{z z}\left(0, z_{n}, z_{n}\right)+C_{z z}\left(0, z_{m}, z_{m}\right)\right]} \\
& \times e^{-q_{\|}^{2} / 2\left[C_{z z}\left(0, z_{n}, z_{n}\right)+C_{z z}\left(0, z_{m}, z_{m}\right)\right]} \\
& \times e^{q_{z}^{2} C_{z z}\left(x_{n}-x_{m}, z_{n}, z_{m}\right)+q_{\|}^{2} C_{z z}\left(x_{n}-x_{m}, z_{n}, z_{m}\right)} .
\end{aligned}
$$

This result takes both the $z$ dependence of the displacement correlation function and the lateral component of the capillary-wave correlation function into account. We come back to the classical equation ${ }^{8}$ for surface scattering with $q_{z} \gg q_{\|}$and neglecting the $z$ dependence of the correlation functions.

The ratio between the vertical and lateral components of the correlation function is given by $\left(q_{z} / q_{\|}\right)^{2}$ meaning that the lateral component is only contributing to the scattering signal when $q_{\|} \geq q_{z}$. This condition is never matched in X-ray reflectivity or transverse rocking scans where data are collected at modest values of $q_{\|}$. However, in GID experiments from liquid surfaces this condition is exactly matched at those positions in reciprocal space where the $q$-dependent surface tension is thought to contribute to the scattering signal. Moreover, in previous analysis of GID experiments it was assumed that the scattering signal is proportional to the Fourier transform of the height-height correlation function. However, according to Eq. (2) only the condition $\left(q_{z}^{2}\right.$ $\left.+q_{\|}^{2}\right) C(x, 0,0)<1$ allows to expand the exponentials in Eq. (2) and guarantees the observation of the Fourier transform $\tilde{C}(q)$ of the correlation functions for the relevant length scales $x$. For example for liquid water we find with $q_{z}$ $=0.3 \AA^{-1}, q_{\|}=0.5 \AA^{-1}$, and $q_{\min }=6 \times 10^{-5} \AA$ a value of $\left(q_{z}^{2}+q_{\|}^{2}\right) C(x, 0,0) \sim 2.0$ implying that the scattering signal is not proportional to $\widetilde{C}(q)$ and the surface Hamiltonian is not directly accessible. Figure 1 shows $\left(q_{z}^{2}+q_{\|}^{2}\right) \sigma^{2}$ (with $q_{z}$ $=0.3 \AA^{-1}$ ) as a function of $q_{\|}^{2}$ for the surface roughness of liquid metals, water, and octamethylcyclotetrasiloxane (OMCTS) as calculated from the respective values of the surface tension. Apparently we find $\left(q_{z}^{2}+q_{\|}^{2}\right) \sigma^{2}>1$ at the rel- 
evant values of the wave-vector transfers for water and OMCTS.

Within the approximation of continuum hydrodynamics the surface tension does not depend on the wave vector, i.e., $H\left(q_{\|}\right)=\gamma q_{\|}^{2}$ and we obtain in the limit $q_{\min } z \ll 1$ an analytical expression for $C_{z z}\left(x, z, z^{\prime}\right)=-\left(k_{B} T / 2 \pi \gamma\right) \ln \left[q_{\min }\left(z+z^{\prime}\right.\right.$ $\left.+\sqrt{x^{2}+\left(z+z^{\prime}\right)^{2}}\right]$. With this the scattering function reads

$$
\begin{aligned}
S(\mathbf{Q})= & 2^{4 \eta} q_{\min }^{\eta} \int_{0}^{\infty} \int_{0}^{\infty} \int_{0}^{\infty}\left(\frac{z \cdot z^{\prime}}{z+z^{\prime}+\sqrt{x^{2}+\left(z+z^{\prime}\right)^{2}}}\right)^{\eta} e^{-\Lambda\left(z+z^{\prime}\right)} \\
& \times e^{i q_{z}\left(z-z^{\prime}\right)} x J_{0}\left(q_{\|} x\right) d x d z d z^{\prime}
\end{aligned}
$$

with $\eta=k_{B} T /(2 \pi \gamma) \cdot\left(q_{\|}^{2}+q_{z}^{2}\right)$. Equation (3) is the static structure factor of capillary waves taking the full-wave-motioninduced static correlations into account and represents the central result of this work. The solution of the integrals in Eq. (3) is however both analytically and numerically a nontrivial task. This is why we discuss here two different approximations: first the vertical damping of capillary waves will be neglected and second the limit of high-surfacetension materials will be discussed.

Neglecting the $z$ dependence of the capillary waves and assuming a constant surface tension $\gamma$ and in the limit $q_{\max }$ $\rightarrow \infty$, the correlation functions can be approximated as $C_{z z}(x, 0,0) \approx\left(-k_{B} T / 2 \pi \gamma\right)\left[\gamma_{e}+\ln \left(q_{\min } x / 2\right)\right] \quad\left(\gamma_{e}\right.$ is Euler's constant) and following Ref. 16 allows arriving at an analytical expressions of the diffuse scattering given by

$$
S(\mathbf{Q})=\frac{2 \pi}{q_{z}^{2}} q_{\min }^{-\eta} e^{-\sigma^{2}\left(q_{z}^{2}+q_{\|}^{2}\right)} \frac{2^{1-\eta}}{q_{\|}^{2-\eta}} \frac{\Gamma(1-\eta / 2)}{\Gamma(\eta / 2)}
$$

with $\eta=k_{B} T /(2 \pi \gamma)\left(q_{z}^{2}+q_{\|}^{2}\right)$, which is valid as long as $\eta<2$. The divergence of the $\Gamma$ function at $\eta=2$ is a direct consequence of the divergence of the logarithmic correlation function in the limit $x \rightarrow 0$. The square of the surface roughness is $\sigma^{2}=\frac{k_{B} T}{2 \pi \gamma} \ln \left(q_{\max } / q_{\min }\right)+\frac{k_{B} T}{2 \pi \gamma} \gamma_{e}$. Note that due to the crude approximation of vanishing $z$ damping, Eq. (4) overestimates the scattered intensity in the vicinity of the critical angle.

Figure 2 shows the scattering signal according to Eq. (4) as a function of parallel wave-vector transfer. Apparently, the signal does not follow the simple $q_{\|}^{-2}$ behavior anymore as soon as $q_{\|} \sim q_{z}$. In this region an excess of scattering intensity can be observed which is due to the more pronounced scattering from the lateral displacement-displacement correlations. At larger wave-vector transfer the increasing DebyeWaller-type factor decreases the scattering intensity rapidly. The inset shows the fraction $F(\mathbf{Q})=S^{\prime}(\mathbf{Q}) / S(\mathbf{Q})$, where $S^{\prime}(\mathbf{Q})$ denotes the small-angle-scattering approximation. The value $F(\mathbf{Q})$ has been interpreted to be $\gamma\left(q_{\|}\right) / \gamma_{0}\left[\gamma_{0}=\gamma\left(q_{\|}\right.\right.$ $=0)]$ previously and it is obvious that Eq. (4) reproduces the overall shape of the reported curves quite well. The excess of scattering intensity in comparison with the small-anglescattering expression $S^{\prime}(\mathbf{Q})$ leads to the minimum in $F(\mathbf{Q})$ in the region $q_{\|} \sim q_{z}$. The final increase in $F(\mathbf{Q})$ is caused by the decreasing intensity of $S(\mathbf{Q})$ due to the Debye-Waller-type factors.

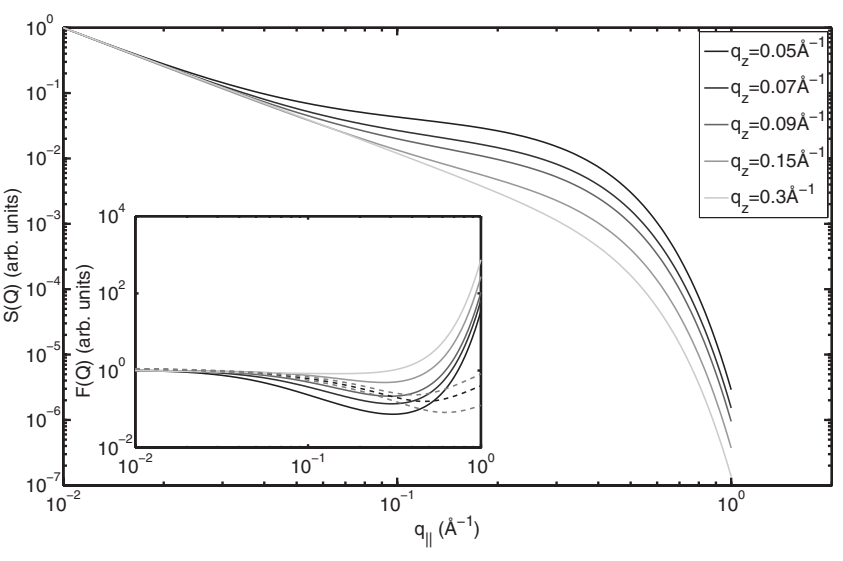

FIG. 2. $S(\mathbf{Q})$ from Eq. (4) plotted for different $q_{z}$ values. The curves are normalized to their first data point. Inset: the fraction $F(\mathbf{Q})=S^{\prime}(\mathbf{Q}) / S(\mathbf{Q})$. The black dashed line shows $F(\mathbf{Q})$ of water obtained by a refinement of scattering data using the small-anglescattering expression given in Ref. 2. The gray dashed lines represent the error channel of the refinement.

For reasons of comparison experimental values of $F(\mathbf{Q})$ for water are shown in the inset of Fig. 2 as a dashed line. The scattering data were taken at the ESRF beamline ID10B and at DELTA BL9 using a similar setup described in Ref. 1. The similarity to $F(\mathbf{Q})$ is clearly visible.

The second approximation of Eq. (3) to be discussed addresses the limit of very-high-surface-tension materials. Here one encounters the case $\left(q_{z}^{2}+q_{\|}^{2}\right) \sigma^{2} \ll 1$ which is for example fulfilled for liquid metals. Let us take the example of liquid gallium which has been measured recently. ${ }^{3}$ With its rather high value of the surface tension $\gamma \sim 0.7 \mathrm{~N} / \mathrm{m}$ we find $\sigma$ $\approx 1 \AA$ which results for the $q$ values of interest in $\left(q_{z}^{2}\right.$ $\left.+q_{\|}^{2}\right) \sigma^{2} \leq 0.5$. With this we can safely expand the exponential in the scattering function (2) and obtain for the diffuse scattering

$$
\begin{aligned}
S(\mathbf{Q})= & \iint d z d z^{\prime} e^{-1 / 2\left(q_{z}^{2}+q_{\|}^{2}\right)\left[C_{x x}(z, z, 0)+C_{z z}\left(z^{\prime}, z^{\prime}, 0\right)\right]} \\
& \times e^{i q_{z}\left(z-z^{\prime}\right)} e^{-\left(z+z^{\prime}\right) / \Lambda}\left[q_{z}^{2} \widetilde{C}_{z z}\left(q_{\|}, z, z^{\prime}\right)+q_{\|}^{2} \widetilde{C}_{x x}\left(q_{\|}, z, z^{\prime}\right)\right],
\end{aligned}
$$

where the penetration depth $\Lambda$ is for liquid metals around 30 A. ${ }^{9}$ Again the problem cannot be solved analytically; therefore, we introduce an effective roughness factor $\sigma_{\text {eff }}$ and write

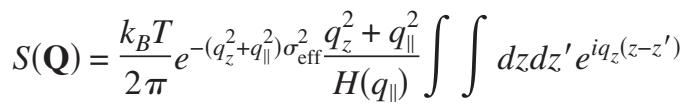

$$
\begin{aligned}
& \times e^{-\left(q_{\|}+1 / \Lambda\right)\left(z+z^{\prime}\right)},
\end{aligned}
$$

which results in

$$
S(\mathbf{Q})=e^{-\left(q_{z}^{2}+q_{\|}^{2}\right) \sigma_{\mathrm{eff}}^{2}} \frac{k_{B} T}{2 \pi H\left(q_{\|}\right)} \frac{q_{\|}^{2}+q_{z}^{2}}{\left(q_{\|}+1 / \Lambda\right)^{2}+q_{z}^{2}} .
$$

Figure 3 shows Eq. (7) for a fixed value of $\sigma_{\text {eff }}$ for different values of the vertical wave-vector transfer $q_{z}$. We find that 


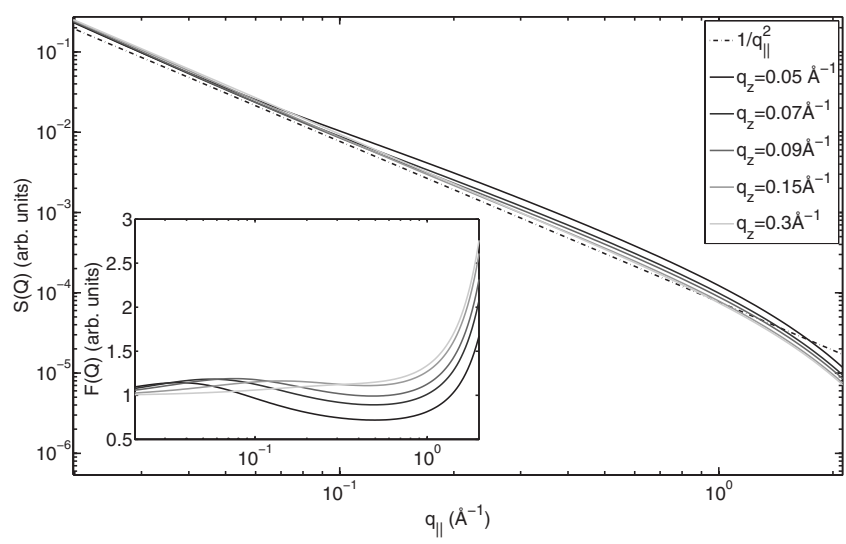

FIG. 3. $S(\mathbf{Q})$ for liquid metals according to Eq. (7). The curves are normalized to their first data point. Inset: the fraction $F(\mathbf{Q})$ $=S^{\prime}(\mathbf{Q}) / S(\mathbf{Q})$.

for small values of $q_{z}$ the scattered intensity exceeds again the $q_{\|}^{-2}$ behavior. This effect decreases with increasing $q_{z}$. The inset shows $F(\mathbf{Q})$ which displays a pronounced $q$ dependence even with the continuum approximation of $H\left(q_{\|}\right)$ $=\gamma_{0} q_{\|}^{2}$. For the smallest $q_{z}$ value the curve first increases with $q_{\|}$before it falls into a minimum at $q_{\|}=0.5 \AA^{-1}$. This minimum is less pronounced with increasing $q_{z}$ and finally vanishes. There is no minimum in the $q_{z}$ region which has been investigated by GID experiments previously, ${ }^{3}$ so one may conclude that the observed minimum is indeed due to a lower surface tension at small length scales. However, a second interpretation seems possible. Suppose that the damping of the capillary waves with decreasing depth in the liquid is less efficient than expected. Introducing a factor $\alpha$ which describes the efficiency of the damping, the penetration depth of the capillary waves is $1 / \alpha q_{\|}$. With this the scattering law is modified to

$$
S(\mathbf{Q})=e^{-\left(q_{z}^{2}+q_{\|}^{2}\right) \sigma_{\mathrm{eff}}^{2}} \frac{k_{B} T}{2 \pi H\left(q_{\|}\right)} \frac{q_{\|}^{2}+q_{z}^{2}}{\left(\alpha q_{\|}+1 / \Lambda\right)^{2}+q_{z}^{2}} .
$$

Figure 4 displays $S(\mathbf{Q})$ and $F(\mathbf{Q})$ from Eq. (8) with different values of the effective damping factor $\alpha$. Apparently, a lower

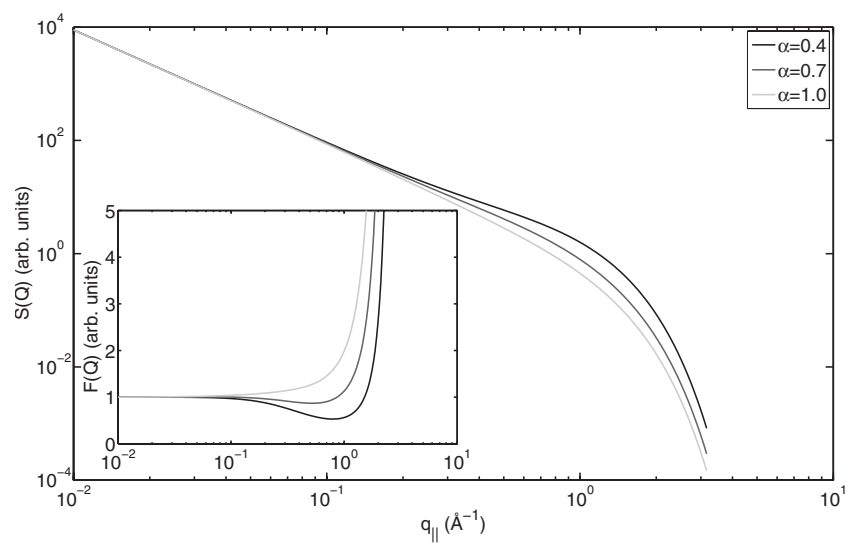

FIG. 4. $S(Q)$ for different values $\alpha$ of the vertical wave damping $\left(q_{z}=0.4 \AA^{-1}\right.$ and $\left.\sigma_{\text {eff }}=0.8 \AA\right)$. The curves are normalized to their first data point. Inset: the fraction $F(\mathbf{Q})=S^{\prime}(\mathbf{Q}) / S(\mathbf{Q})$.

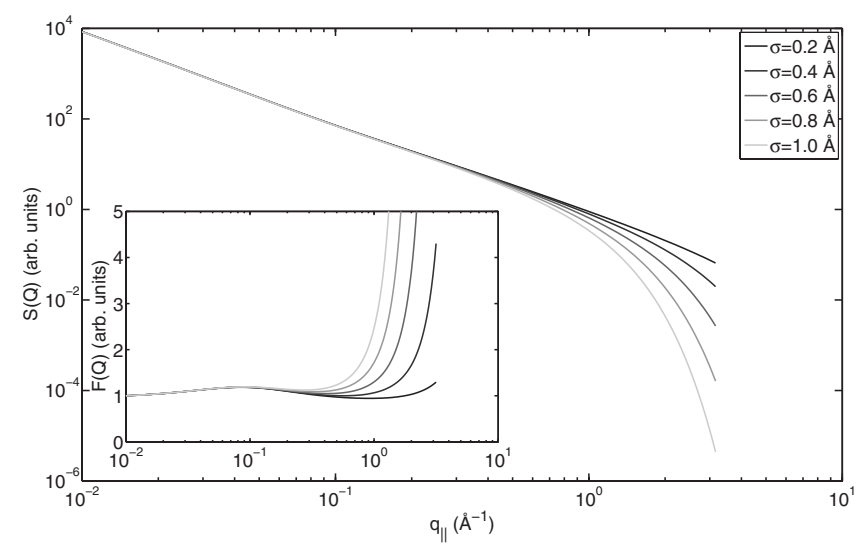

FIG. 5. $S(\mathbf{Q})$ for liquid metals with different values of the effective surface roughness $\sigma_{\text {eff }}$ for $q_{z}=0.1 \AA^{-1}$. The curves are normalized to their first data point. Inset: the fraction $F(\mathbf{Q})$ $=S^{\prime}(\mathbf{Q}) / S(\mathbf{Q})$.

value of the depth damping of the capillary waves leads to a minimum in $F(\mathbf{Q})$ at higher values of $q_{z}$. For example a value of $\alpha=0.7$ increases the penetration depth of the capillary waves at $q_{\|}=0.1 \AA^{-1}$ from 10 to $14 \AA$ and at $q_{\|}$ $=0.3 \AA^{-1}$ from 3.3 to $4.7 \AA$. So for $q_{\|}=0.3 \AA^{-1}$ the length scale probed in the direction parallel to the surface is still large with $20 \AA$ while the length scales probed in the vertical direction is already at the values of single atoms.

The exact shape of $S(\mathbf{Q})$ is also influenced by the value of the effective roughness. Figure 5 displays $S(\mathbf{Q})$ and $F(\mathbf{Q})$ for a fixed value of $q_{z}=0.4 \AA^{-1}$ with different values of the effective roughness $\sigma_{\text {eff. }}$. Obviously, the increase in $F(\mathbf{Q})$ at large $q_{\|}$values strongly depends on the value of the effective roughness. A large $\sigma_{\text {eff }}$ reduces the scattered intensity via the Debye-Waller-type factor and in turn leads to a significant increase in $F(\mathbf{Q})$ at large $q_{\|}$. Both approximations to Eq. (3) show that the static scattering cross section of capillary waves at large momentum transfers parallel to the surface is different from the small-angle-scattering expression used previously to analyze GID data from liquid surfaces.

\section{SUMMARY}

For liquids with low surface tensions such as, e.g., water the scattering cross section and consequently the scattered intensity in a GID measurement at large values of $q_{\|}$is not proportional to the Fourier transform of the height-height correlation function. The surface Hamiltonian is thus not directly accessible with surface-scattering experiments for those liquids and the existence of a wave-vector-dependent surface tension cannot be claimed. In the case of liquid metals the scattering signal is a mixture of height and lateral fluctuations and the surface Hamiltonian is in principle directly accessible. However, the exact shape of $H\left(q_{\|}\right)$strongly depends on the parameters used in the analysis such as surface roughness and the depth damping of the capillary waves. Our results have implications for the interpretation of $\mathrm{x}$-ray scattering data from all soft matter interfaces if an understanding in the nanometer regime is desired. 


\section{ACKNOWLEDGMENTS}

The authors acknowledge the ESRF for providing synchrotron radiation facilities and the DELTA machine group for providing the synchrotron radiation and technical support.

\section{APPENDIX: DISPLACEMENT-DISPLACEMENT CORRELATION FUNCTIONS}

The displacement-displacement correlation function is defined as $C_{\alpha, \beta}\left(z, z^{\prime}, x\right)=\left\langle u_{\alpha}(z, 0) u_{\beta}\left(z^{\prime}, x\right)\right\rangle$, where $x$ is the coordinate parallel to the surface and $z$ perpendicular to the liquid surface. Explicit expressions for the correlation functions have been obtained with the help of linear-response theory ${ }^{14,15}$ yielding the dynamic susceptibilities $\chi_{\alpha \beta}\left(q_{\|}, z, z^{\prime}, \omega\right)$. The fluctuation dissipation theorem then allows calculating the static displacement-displacement correlation function via the real part $\mathfrak{R}$ of the static susceptibility: $C_{\alpha, \beta}\left(z, z^{\prime}, x\right)=k_{B} T \int d q_{\|}^{2} e^{i q_{\|} x} \Re \chi_{\alpha \beta}\left(q_{\|}, z, z^{\prime}, \omega=0\right)$.

For the case $z=z^{\prime}=0$ we find

$$
C_{x, x}(0,0, x)=C_{z, z}(0,0, x)=\frac{k_{B} T}{2 \pi \gamma} \int_{0}^{\infty} d q \frac{q}{q_{g}^{2}+q^{2}} J_{0}(q x)
$$

$$
=\frac{k_{B} T}{2 \pi \gamma} K_{0}\left(q_{g} x\right),
$$

where $K_{0}$ is the modified Bessel function of the second kind. $q_{g}^{2}=\rho g / \gamma$ is the gravitational cutoff with $\rho$ being the density of the liquid and $g$ the gravitational constant. This result has some useful properties for calculating the x-ray scattering cross section. However, due to the limit $K_{0}\left(q_{g} x\right) \rightarrow \infty$ for $x$ $\rightarrow 0$ the scattering cross section contains singularities in reciprocal space. ${ }^{17}$ The singularities can be avoided by introducing an upper limit $q_{\max }$ within the integral ${ }^{17}$

$$
C_{x, x}(0,0, x)=C_{z, z}(0,0, x)=\frac{k_{B} T}{2 \pi \gamma} \int_{0}^{q_{\max }} d q \frac{q}{q_{g}^{2}+q^{2}} J_{0}(q x)
$$

$$
\begin{aligned}
= & \frac{k_{B} T}{2 \pi \gamma}\left(\frac{q_{\max }}{q_{g}}\right)^{2} \sum_{k=0}^{\infty} \frac{(-1)^{k}}{\Gamma(1+k)^{2}} F_{1} \\
& \times\left(1 ; 1+k ; 2+k ;-q_{\max }^{2} / q_{g}^{2}\right)\left(\frac{q_{\max } x}{2}\right)^{2 k},
\end{aligned}
$$

where ${ }_{2} F_{1}$ is a regularized hypergeometric function.

For the case $z, z^{\prime} \neq 0$ we approximate the correlation function according to

$$
C_{x, x}\left(z, z^{\prime}, x\right)=C_{z, z}\left(z, z^{\prime}, x\right)=\frac{k_{B} T}{2 \pi \gamma} \int_{q_{\min }}^{\infty} d q \frac{J_{0}(q x)}{q} e^{-q\left(z+z^{\prime}\right)}
$$

$$
\begin{aligned}
= & -\frac{k_{B} T}{2 \pi \gamma} \ln \left[q_{\min }\left(z+z^{\prime}+\sqrt{x^{2}+\left(z+z^{\prime}\right)^{2}}\right]\right. \\
& +C+O\left[q_{\min }\left(z+z^{\prime}\right)\right] \\
\approx & -\frac{k_{B} T}{2 \pi \gamma} \ln \left[q_{\min }\left(z+z^{\prime}+\sqrt{x^{2}+\left(z+z^{\prime}\right)^{2}}\right],\right.
\end{aligned}
$$

which is valid as long as $q_{\min }\left(z+z^{\prime}\right) \ll 1$.
${ }^{1}$ C. Fradin, A. Breslau, D. Luzet, D. Smilgies, M. Alba, N. Boudet, K. Mecke, and J. Daillant, Nature (London) 403, 871 (2000).

${ }^{2}$ S. Mora, J. Daillant, K. Mecke, D. Luzet, A. Braslau, M. Alba, and B. Struth, Phys. Rev. Lett. 90, 216101 (2003).

${ }^{3}$ D. Li, B. Yang, B. Lin, M. Meron, J. Gebhardt, T. Graber, and S. A. Rice, Phys. Rev. Lett. 92, 136102 (2004).

${ }^{4}$ D. Li, X. Jiang, B. Lin, M. Meron, and S. A. Rice, Phys. Rev. B 72, 235426 (2005).

${ }^{5}$ M. K. Sanyal, S. K. Sinha, K. G. Huang, and B. M. Ocko, Phys. Rev. Lett. 66, 628 (1991).

${ }^{6}$ O. Shpyrko, M. Fukuto, P. Pershan, B. Ocko, I. Kuzmenko, T. Gog, and M. Deutsch, Phys. Rev. B 69, 245423 (2004).

${ }^{7}$ K. R. Mecke and S. Dietrich, Phys. Rev. E 59, 6766 (1999).

${ }^{8}$ S. K. Sinha, E. B. Sirota, S. Garoff, and H. B. Stanley, Phys. Rev. B 38, 2297 (1988).
${ }^{9}$ M. Tolan, X-ray Scattering from Soft Matter Thin Films, Springer Tracts in Modern Physics Vol. 148 (Springer, Berlin, 1999).

${ }^{10}$ G. H. Vineyard, Phys. Rev. B 26, 4146 (1982).

${ }^{11} \mathrm{~J}$. Als-Nielsen and D. McMorrow, Elements of Modern X-ray Physics (Wiley, New York, 2001).

${ }^{12}$ V. G. Levich, Physicochemical Hydrodynamics (Prentice-Hall, Englewood Cliffs, NJ, 1962).

${ }^{13}$ M. A. Bouchiat and J. Meunier, J. Phys. (France) 32, 561 (1971).

${ }^{14}$ S. Mora and J. Daillant, Eur. Phys. J. B 27, 417 (2002).

${ }^{15}$ R. Loudon, Proc. R. Soc. London, Ser. A 372, 275 (1980).

${ }^{16}$ P. Dutta and S. K. Sinha, Phys. Rev. Lett. 47, 50 (1981).

${ }^{17}$ T. Ghaderi, Ph.D. thesis, University of Dortmund, Germany, 2006; http://eldorado.uni-dortmund.de:8080/handle/2003/22293 